\title{
Effect of Carbon Nanofiber Heat Treatment on Physical Properties of Polymeric Nanocomposites-Part I
}

\author{
Khalid Lafdi, William Fox, Matthew Matzek, and Emel Yildiz \\ University of Dayton, 300 College Park, Dayton, OH 45469, USA
}

Received 22 March 2007; Accepted 2 June 2007

Recommended by Donglu Shi

The definition of a nanocomposite material has broadened significantly to encompass a large variety of systems made of dissimilar components and mixed at the nanometer scale. The properties of nanocomposite materials also depend on the morphology, crystallinity, and interfacial characteristics of the individual constituents. In the current work, vapor-grown carbon nanofibers were subjected to varying heat-treatment temperatures. The strength of adhesion between the nanofiber and an epoxy (thermoset) matrix was characterized by the flexural strength and modulus. Heat treatment to $1800^{\circ} \mathrm{C}$ demonstrated maximum improvement in mechanical properties over that of the neat resin, while heat-treatment to higher temperatures demonstrated a slight decrease in mechanical properties likely due to the elimination of potential bonding sites caused by the elimination of the truncated edges of the graphene layers. Both the electrical and thermal properties of the resulting nanocomposites increased in conjunction with the increasing heat-treatment temperature.

Copyright (c) 2007 Khalid Lafdi et al. This is an open access article distributed under the Creative Commons Attribution License, which permits unrestricted use, distribution, and reproduction in any medium, provided the original work is properly cited.

\section{INTRODUCTION}

Research on vapor-grown carbon fibers has been heightened in recent years by the discovery of carbon nanotubes. Such fibers are characterized by an extraordinarily high tensile modulus, tensile strength, and high electrical and thermal conductivity. A decrease in the diameter of a vaporgrown carbon fiber causes gradual improvement in mechanical properties. As the fiber diameter reaches the threshold value of $1 \mu \mathrm{m}$, distinguishing the transition from a fiber to a nanofiber, the improvement in mechanical properties becomes more significant [1]. Vapor-grown carbon nanofibers can be prepared with diameters ranging from $15 \mathrm{~nm}$ to $100 \mathrm{~nm}$. These fibers are continuous and have hollow cores. Their morphology resembles that of multiwall carbon nanotubes. Nanofibers can have a number of different internal structures, wherein graphene layers are arranged as concentric cylinders, nested truncated cones, segmented structures, or stacked coins [2]. External morphologies include kinked and branched structures and diameter variation. The percolation threshold for carbon nanofibers is low indicating that only a small amount of nanofiber additive is required for conducting filler applications. The intrinsic stiffness and strength of carbon nanofibers, combined with these superior transport properties, present the opportunity to develop multifunctional nanofiber composites with tailored physical and mechanical properties.

Graphitization is an effective method of removing defects from carbon nanofibers that diminish their electrical and mechanical properties. The structure of the initial carbon generally determines the physical properties of graphitized carbon materials due to the thermally activated kinetic process of graphitization. It is important to understand graphitization behavior for carbon nanofibers because structural changes, purification, and enhanced structural perfection by heat-treatment may improve a specific application of the nanofibers.

Endo et al. [3] heat treated cup-stacked-type carbon nanofibers from 1800 to $3000^{\circ} \mathrm{C}$ to examine structural changes. The truncated cones cause a high chemical reactivity in the outer surface and the inner hollow cores because these end planes of graphene layers are active edge sites. Heat treatment to $3000^{\circ} \mathrm{C}$ resulted in transformation to a rugged surface and the formation of energetically stable loops between adjacent graphene layers from the unstable edge planes in both the outer surface and the inner hollow cores. Examination of the fibers by X-ray diffraction and Raman spectroscopy identified that the interlayer spacing of the graphitized samples increased possibly due to the large number of loop formations between adjacent 
graphene layers. The absence of separation of (101) and (100) lines and low intensity of (004) lines indicated that the nanofibers achieved relatively low graphitizatioin following heat-treatment to $3000^{\circ} \mathrm{C}$. The formation of loops began below $2100^{\circ} \mathrm{C}$ and was followed by few changes up to $3000^{\circ} \mathrm{C}$ on the outer surface of the carbon nanofibers. With increasing heat-treatment temperature, there was a progressive decrease in the electrical resistivity in the bulk state for carbon nanofibers due to the amount of loops, especially on the outer surface of the carbon nanofibers. There was a significant decrease in the electrical resistivity from the as-grown nanofiber to the nanofiber heat treated to $1800^{\circ} \mathrm{C}$ due to the evolution of volatile material.

Lim et al. studied stacked-coin-type nanofibers and the impact of mechanical and chemical treatments on the morphology of the fibers [4]. Heat treatment at $2800^{\circ} \mathrm{C}$ induced closed loop ends on the surface of the nanofibers formed by folding of some planar hexagons at their edges. The heattreatment removed $\mathrm{C}-\mathrm{H}$ bonds and densely stacked hexagonal layers of graphene, forming chemically active sites on the edges. The edges were stabilized by bonding to each other, even though the bonding caused high tension through the formation of a sharp curvature. Acidic oxidation of the nanofibers cuts off the closed loop ends, resulting in improved overall alignment of graphene layers. Both treatments generated many free edges and a high graphitization extent, indicating the possibility of improved interfacial bonding with a polymer matrix.

Katayama et al. examined the effect of heat-treatment on multiwall carbon nanotubes with bamboo-like structures [5]. Heat treatment at $2800^{\circ} \mathrm{C}$ reduced the interlayer spacing to that of graphite. In addition, encapsulates in the bamboostructure with a small diameter were opened, and metal impurities were entirely removed from the nanotube. The decrease in interlayer spacing lies in contrast to the observance reported by Endo et al. for carbon nanofibers heat treated to $3000^{\circ} \mathrm{C}$.

Kiselev et al. examined by high-resolution electron microscopy the structural changes to multiwall carbon nanotubes following heat-treatment [6]. Links were formed between the neighboring open edges by loops along the tube sides on both the insides and outsides of the nanotubes. The radii of curvature of the loops were noted at $1.05-1.40 \mathrm{~nm}$ which is close to the diameter of single-wall nanotubes. The degree of carbon layer linking was dependent on the temperature of the heat-treatment. After treatment at $1200^{\circ} \mathrm{C}$, only a small amount of linked layers were observed along the external sides. At treatment above $2000^{\circ} \mathrm{C}$, the number of loops per unit volume increased and loops were observed on both the external and internal surfaces.

A number of results have been reported on the effect of heat-treatment of carbon nanofibers on nanocomposite properties. $\mathrm{Xu}$ et al. included nanofiber/vinyl ester composite with nanofibers heat treated to $3000^{\circ} \mathrm{C}$ in an examination of electrical properties of nanocomposites [7]. The percolation threshold was found to be between 2 and $3 \mathrm{wt} \%$ fiber loading. The heat-treated fibers were better electrical conductors than pyrolytically stripped fibers. The higher surface activity of the heat-treated fibers should lead to thicker resin coating and more complete resin infusion into the fibers during mixing. However, the measured flexural modulus of the nanocomposite was equal to that of the pure resin. This may have been due to poor fiber dispersion in the matrix. In addition, the measured glass transition temperature of a nanocomposite with heat-treated fibers was nearly $20^{\circ} \mathrm{C}$ higher than that of a nanocomposite with pyrolitycally stripped fibers.

Finegan et al. [8] noted that more graphitic fibers of higher graphitization index tend to make composites having both lower strength and modulus. Graphitized fibers projected much further from the fracture surface of a nanofiber/polypropylene composite than air-etched fibers implying that the interfacial shear strength of the graphitized fibers was significantly lower. The polymer did not appear to wet the fiber surface in either cases, indicating that low interfacial strength is not singularly dependent on surface wetting. The results from this study may have been impacted by ball milling of all nanofiber samples resulting in lower fiber aspect ratios.

Kuriger et al. examined the thermal and electrical properties of heat treated nanofiber/polypropylene composites [9]. The electrical resistivity decreased with fiber volume fraction. This is characteristic of the electrical conductivity of composites which is governed by percolation and quantum mechanical tunneling between the fibers. The electrical resistivity was significantly lower than glass fiber reinforced polymers. The thermal conductivity increased with fiber loading, and reached a maximum with $23 \%$ fiber volume fraction at $5.38 \mathrm{~W} / \mathrm{mK}$ in the longitudinal direction.

Ma et al. included heat-treated nanofiber/polyester composites in their study of nanocomposite properties. Thermogravimetric analysis showed that the onset of degradation for nanofibers heat treated at $3000^{\circ} \mathrm{C}$ was nearly $700^{\circ} \mathrm{C}$ compared to approximately $300^{\circ} \mathrm{C}$ for as-grown nanofibers. The tensile modulus of the nanocomposite was slightly higher than that of the neat resin, and the tensile strength was comparable to the neat resin and significantly higher than its pyrolytically stripped-fiber nanocomposite counterpart. The inability of the heat treated fibers to improve the tensile properties of the neat resin may be due to the graphitic planes not being aligned parallel to the nanofiber axis. This counters the alignment of the nanofibers in the neat resin. In addition, the heat-treated nanofibers were ball milled leading to attrition of the nanofiber aspect ratio, which is detrimental to the tensile properties. The compressive strength and torsional modulus of the heat-treated nanofiber composite were significantly higher than that of the neat resin. The nanofibers may have acted as a barrier to halt propagation, thereby improving compressive properties.

In this study, carbon nanofibers were subjected to heattreatment temperatures ranging from $1500^{\circ} \mathrm{C}$ to $3000^{\circ} \mathrm{C}$ to alter the properties of the reinforcement and the physical and chemical interactions between the nanofiber and the epoxy polymer. The strength of adhesion between the fiber and an epoxy (thermoset) matrix was characterized by the flexural strength and modulus, and the electrical and thermal properties of the composites were investigated from the viewpoint of heat-treatment temperature of the carbon nanofibers. 


\section{EXPERIMENTAL SETUP}

The carbon nanofibers used in this study were produced at Applied Sciences Inc. and were from the Pyrograf III family of fibers. This group of nanofibers (labeled PR-24) has diameters between 60 and $100 \mathrm{~nm}$ and lengths ranging from 30 to 100 microns.

The nanofibers were heat treated in an atmospherecontrolled batch furnace. Approximately $300 \mathrm{~g}$ of nanofibers were placed in a ceramic crucible for the heat-treatment. The furnace was purged with nitrogen gas for one hour prior to heating. The heating rate was $100^{\circ} \mathrm{C}$ per hour, and the furnace was held at the target temperature for one hour prior to cooling. The target temperatures were $1500,1800,2000$, and $3000^{\circ} \mathrm{C}$. The carbon nanofibers were dispersed within the epoxy resin at loading rates of four, eight, and twelve percent by weight. Previous work [10] indicated that loading beyond twelve percent by weight actually lead to a decrease in nanocomposite mechanical properties. In addition, processing nanocomposites with loads beyond twelve percent by weight becomes difficult in establishing a homogenous mixture. The as-received carbon nanofibers, designated as PS, and the neat resin were used as a baseline for the study.

The mechanical properties of the nanocomposites were measured using the three-point flexural strength test according to ASTM D790-00. Thermal diffusivity of each nanocomposite incorporated a xenon flash diffusivity test. The thermal diffusivity parameter was used in conjunction with the specific heat and density of the nanocomposite to calculate the thermal conductivity. The tests for specific heat and density were carried out according to ASTM E1269-89 and ASTM C693-74, respectively. The electrical resistivity of each nanocomposite was tested utilizing a four-point test according to ASTM B193-87.

\section{RESULTS AND DISCUSSION}

In order to fully understand the properties that make carbon nanofibers unique, both the microstructure and surface of nanofibers samples were characterized. The pristine and surface-treated nanofibers consist of a variety of carbon configurations: helical, straight, nested, and carbon blacks with narrow distribution of diameters and lengths (Figure 1). However, two main configurations seem to be dominant within the nanoconstituents: nested and straight carbon nanofibers.

The five major carbon constituents represented under the term carbon nanofibers are graphically represented in Figure 2 to better depict their physical structures. Carbon blacks are nanometric spheres of agglomerated carbon also recognized as soot. Helical carbon nanofibers, also known as nanocoils, consist of carbon nanofibers that have a configuration similar to that of a DNA strand. A major constituent of the carbon nanofibers is straight carbon nanofibers, which exist as a series of coaxial carbon cylinders surrounding a central hollow tube. The dominant species within carbon nanofibers include bamboo and nested carbon nanofibers. The bamboo species are similar to that of the straight carbon nanofibers, except that they are segmented along their

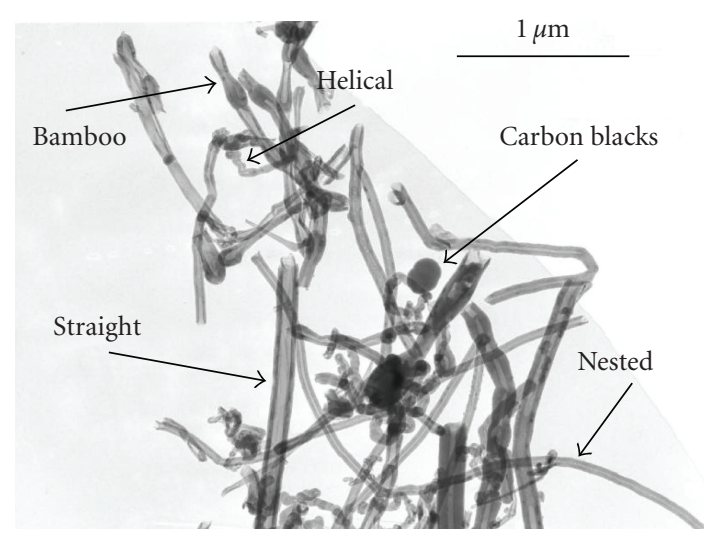

FIGURE 1: Bright-field image of pristine carbon nanofibers (PR-24).

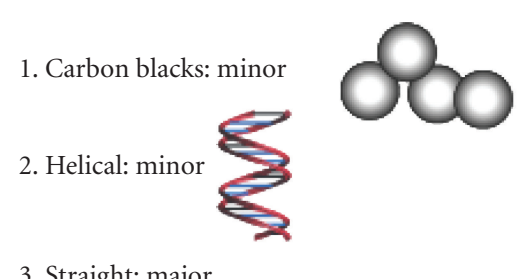

3. Straight: major

3. Nested (DC): dominant

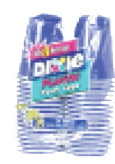

4. Bamboo: dominant

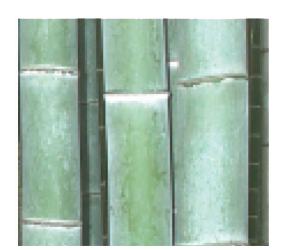

Figure 2: Models of various nanofiber configurations.

lengths. Nested carbon nanofibers have an orientation similar to that of a set of stacked Dixie cups with a hollow core, and are also referred to as fishbone-type carbon nanofibers [11].

At low temperatures, carbon exhibits only local molecular ordering. As they are heat treated, an increase in temperature results in the aromatic molecules becoming stacked in a column structure. Further heat-treatment causes these columns to coalesce forming a distorted, wavy structure. After surpassing a temperature of $2500^{\circ} \mathrm{C}$, the distorted graphene layers of carbon become flattened, forming an aligned structure. If the material is graphitic, it will attain the minimum interlayer spacing in the graphite order between graphene layers (Figure 3).

By analogy, after heat treating the pristine nanofibers to a temperature of $3000^{\circ} \mathrm{C}$, graphene layers became straight, and minimum interlayer spacing was reached for the PR-24 HHT. As shown by a TEM micrograph (Figures 4 and 5), the layers 


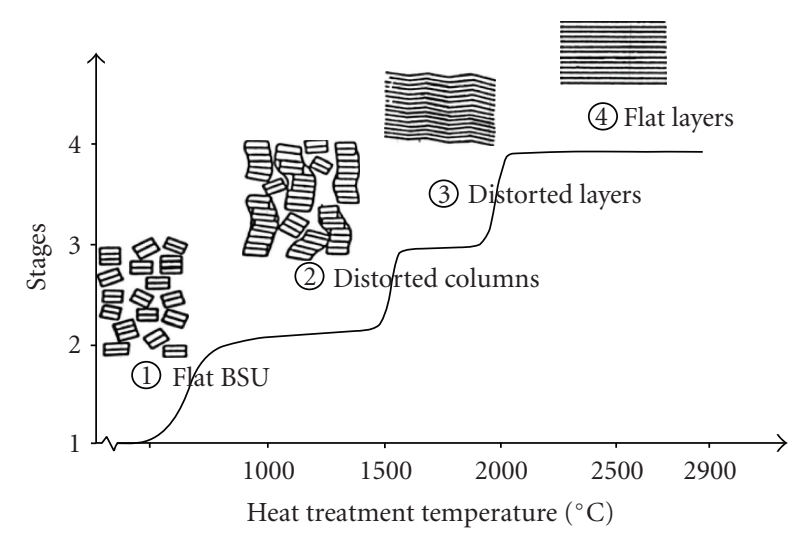

FIGURE 3: Carbon plane structure as a function of heat-treatment temperature.

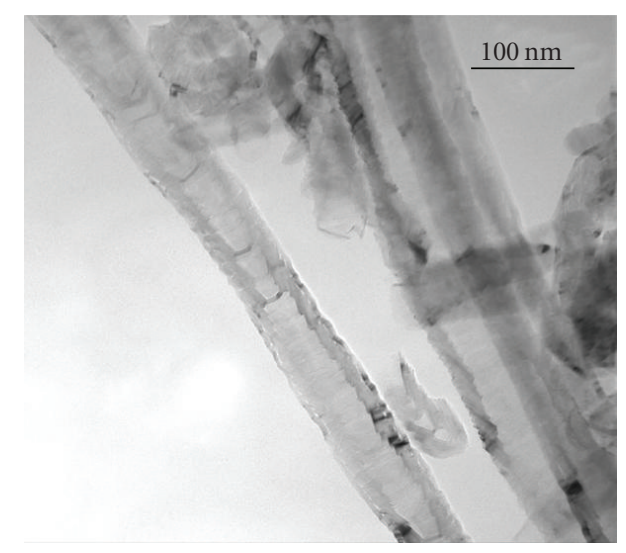

Figure 4: Bright-field micrograph of "Dixie cup" carbon nanofiber structure.

within the "Dixie cup" carbon nanofiber have coalesced following heat-treatment.

At this magnification, the inclination angle of each "cup" is apparent. Within each cup, it can be seen that the localized ordering of the graphene planes has been changed due to coalescence resulting in continuous planes. The stacking effect is shown through the use of a grey scale. The walls of the nanofibers are dark due to their high electronic density. The surrounding regions are starkly lighter with low electronic densities.

At high magnification, the graphene layers appear very straight without any disclination defects. However, there is no change in the inclination angle to the central core axis. The edge of any pair of graphene layers has been rounded encapsulating carbon planes' exposed edges. This allows the exposed graphene planes to attain a level of maximum structural stability.

The improvements in mechanical properties are shown in Figures $6 \& 7$. The flexural modulus increased with increasing nanofiber load. The maximum modulus of $3.5 \mathrm{GPa}$, was achieved for the nanocomposite sample with twelve weight percent loading of nanofibers heat treated to $1800^{\circ} \mathrm{C}$.

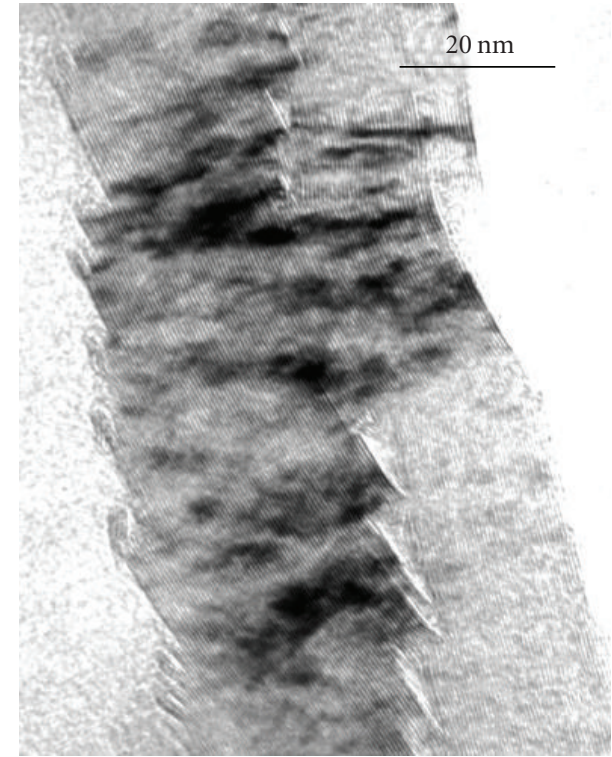

FIGURE 5: High-resolution imaging of localized area of "Dixie cups" structure.

With increasing heat-treatment temperature, the modulus for twelve weight percent loading of nanofibers heat treated to $2000^{\circ} \mathrm{C}$ and $3000^{\circ} \mathrm{C}$ decreased to 3.1 and $3.0 \mathrm{GPa}$ respectively. This indicated that with increasing the amount of graphitization, the adhesion between the carbon nanofiber and the epoxy resin decreased. The increased graphitization of the nanofibers may cause the previously truncated graphene layers to loop together, thereby eliminating free edges for bonding with the polymer matrix. However, the heat-treatment of the carbon nanofibers did show an improvement over the pristine nanofibers. For the as-received PS nanofibers, the modulus at twelve weight percent loading was $2.6 \mathrm{GPa}$. This increase in modulus may be due to the removal of "dirt" - polyaromatic hydrocarbons, sulfur, and oxygen- and nitrogen-based functional groups-allowing for free ends of the graphene sheets to bind with the epoxy.

The ultimate strength of the nanocomposites decreased with increasing nanofiber load for the heat treated nanofiber composites. Again, the nanofibers heat treated to $1800^{\circ} \mathrm{C}$ exhibited the highest strength values for the heat-treated samples at all nanofiber loading levels. Heat treatment had a positive effect on strength for low (four weight percent) nanofiber loading; however at higher nanofiber loadings (eight and twelve weight percent), heat-treatment of nanofibers actually lead to a lower strength than the pristine PS nanofiber composites. The minimal improvements in strength compared to that of modulus may be an indication that the chemical adhesion between the nanofiber and the epoxy matrix is not optimized. This can be improved through chemical functionalization of the nanofiber. In addition, strength of the nanocomposite could be improved in one direction by alignment of the nanofibers in the matrix. The improvement in electrical and thermal properties of the nanocomposites comprised of heat treated nanofibers was 


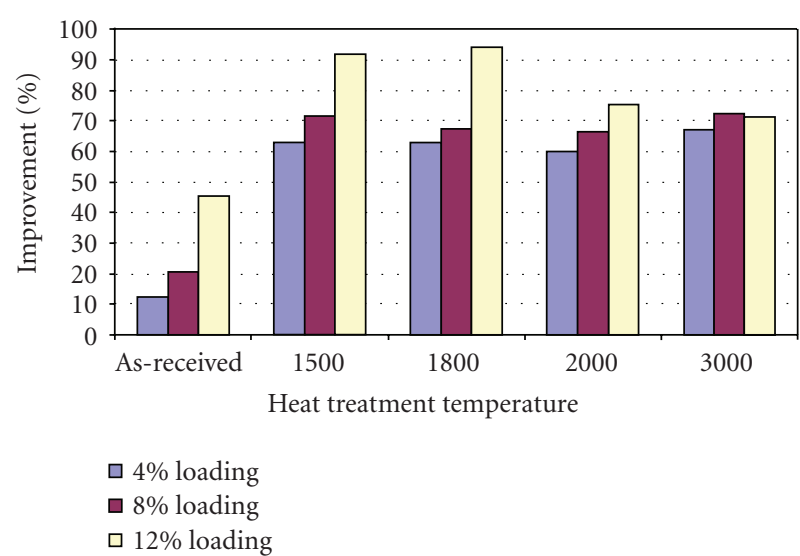

Figure 6: Nanocomposite flexural modulus percent improvement over neat resin as a function of nanofiber heat-treatment temperature.

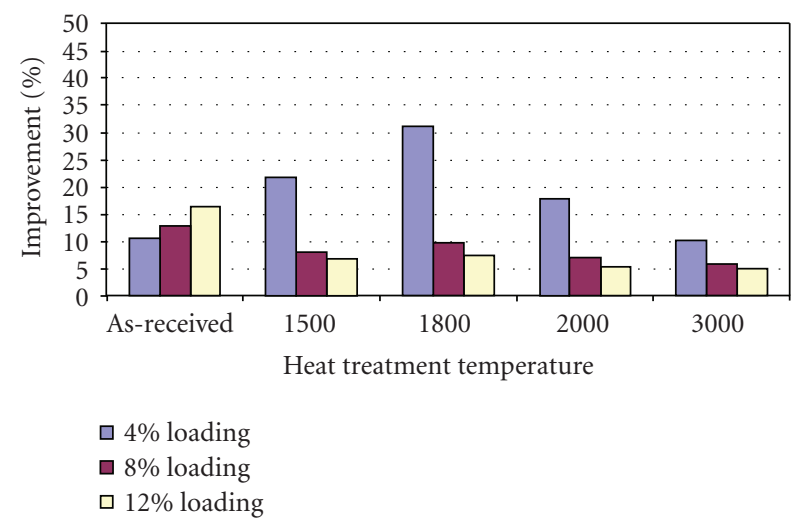

FIGURE 7: Nanocomposite flexural strength percent improvement over neat resin as a function of nanofiber heat-treatment temperature.

significant. This drastic improvement of thermal and electrical properties of the high-heat-treated nanofiber-based composite over that of the PS nanofiber-based composite is due to the structural changes that occur during heat-treatment. The alignment of the graphene layers within the nanofibers allows for a more efficient transfer of phonons and electrons. The improvement in electrical resistivity is shown in Figure 8. While the addition of pristine PS nanofibers at four weight percent loading decreased the resistivity of the nanocomposite by five orders of magnitude over the neat epoxy resin, heat-treatment of the nanofibers to $1500^{\circ} \mathrm{C}$ reduced the resistivity by eight orders of magnitude and heattreatment of the nanofibers to $3000^{\circ} \mathrm{C}$ decreased the resistivity by nine orders of magnitude. In addition, with increasing heat-treatment temperature, the electrical resistivity decreased-from $440 \Omega \cdot \mathrm{cm}$ at four weight percent loading of nanofibers heat treated to $1500^{\circ} \mathrm{C}$ to $94 \Omega \cdot \mathrm{cm}$ at four weight percent loading of nanofibers heat treated to $3000^{\circ} \mathrm{C}$. Increasing the nanofiber loading further decreased the elec-

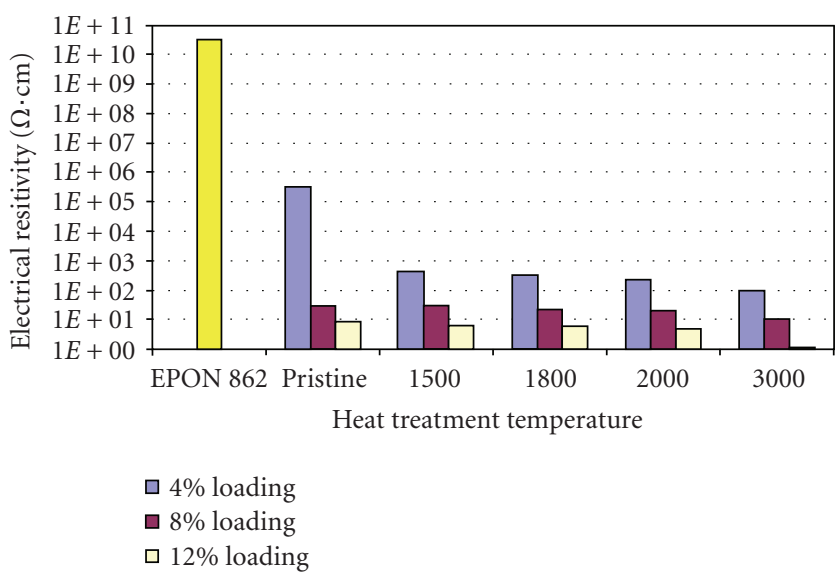

FIgURE 8: Nanocomposite resistivity as a function of nanofiber heat-treatment temperature.

trical resistivity. The electrical resistivity of the nanocomposite with twelve weight percent loading of nanofibers heat treated to $1500^{\circ} \mathrm{C}$ was $6.5 \Omega \cdot \mathrm{cm}$, and the electrical resistivity of the nanocomposite with twelve weight percent loading of nanofibers heat treated to $3000^{\circ} \mathrm{C}$ was $1.2 \Omega-\mathrm{cm}$.

In a similar manner, the thermal conductivity of neat epoxy resin increased significantly with the addition of nanofibers (Figure 9). While the addition of as-received PS nanofibers at four and twelve weight percent loadings increased the thermal conductivity of the neat resin by $21 \%$ and $105 \%$, respectively, the heat-treatment of the nanofibers to $1500^{\circ} \mathrm{C}$ increased the thermal conductivity by $64 \%$ at four weight percent loading and 194\% at twelve weight percent loading. Heat treatment of the nanofibers to $3000^{\circ} \mathrm{C}$ increased the thermal conductivity by $133 \%$ at four weight percent loading and $6191 \%$ at twelve weight percent loading. The degree of alignment and reduction in the interlayer spacing between graphene layers leads to a great increase in thermal conductivity and eventually to low electrical resistivity.

\section{CONCLUSIONS}

The heat-treatment of carbon nanofibers led to the removal of impurities from the nanofiber and resulted in altered physical properties of a nanofiber-reinforced epoxy composite. During heat-treatment, the structure within the carbon nanofibers is altered from local molecular ordering to that of coalesced, flattened graphene layers. Heat treatment up to $1800^{\circ} \mathrm{C}$ resulted in improved flexural modulus and strength of the nanocomposite. Additional heat-treatment to higher temperatures led to increased conversion to graphene layers and resulted in lower mechanical properties due to poor adhesion to the epoxy matrix caused by the elimination of the free truncated edges of the graphene layers available to bond to the polymer matrix. The alignment of graphene layers within the nanofibers allowed for a more efficient transfer of phonons and electrons resulting in significant decrease in electrical resistivity and increase in thermal conductivity 


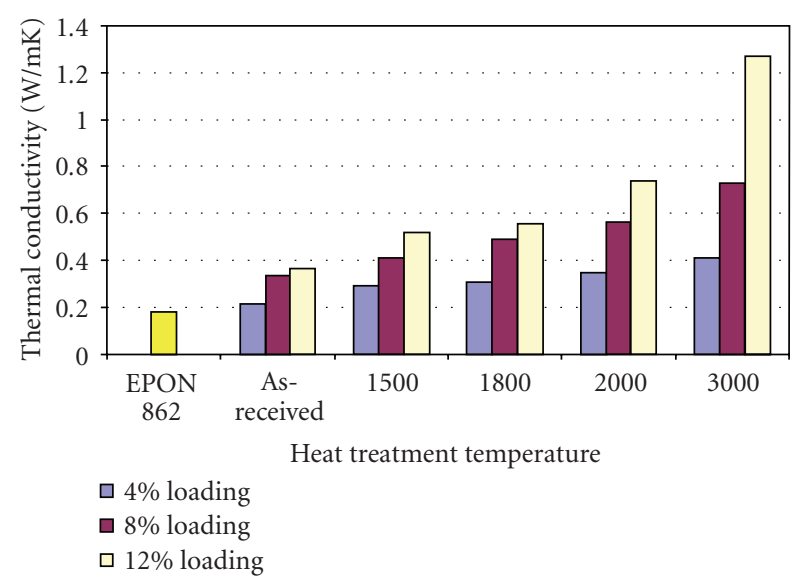

FIgURE 9: Nanocomposite thermal conductivity as a function of nanofiber heat-treatment temperature.

of the neat epoxy resin. Heat treatment of the nanofibers allowed for the attainment of superior electrical and thermal properties at low fiber loadings that are not possible with pristine pyrolytically stripped carbon nanofibers.

The testing of heat-treated nanofiber-based nanocomposites demonstrated that the resulting nanocomposite properties must be compromised. If the end result of the nanocomposite is a desired high mechanical property, the nanofibers should not be heat treated above $1800^{\circ} \mathrm{C}$. However, if the end result is a desired high electrical or thermal property, the nanofiber must be heat treated to $3000^{\circ} \mathrm{C}$. At this higher temperature, while the graphene layers are aligned with minimal interlayer spacing to allow efficient electron and phonon transfer, the ends of the graphene planes tend to loop together and reduce the number of available sites for bonding with the resin. Therefore, a better method for improving interfacial adherence may be to chemically functionalize the nanofiber surface. This would impart groups on the surface that would bind to the resin without changing the structure of the nanofiber resulting in reduced chemical bonding sites.

\section{REFERENCES}

[1] V. Z. Mordkovich, "Carbon nanofibers: a new ultrahigh-strength material for chemical technology," Theoretical Foundations of Chemical Engineering, vol. 37, no. 5, pp. 429-438, 2003.

[2] B. Maruyama and K. Alam, "Carbon nanotubes and nanofibers in composite materials," SAMPE Journal, vol. 38, no. 3, pp. 59-70, 2002.

[3] M. Endo, Y. A. Kim, T. Hayashi, et al., "Microstructural changes induced in "stacked cup" carbon nanofibers by heat treatment," Carbon, vol. 41, no. 10, pp. 1941-1947, 2003.

[4] S. Lim, S.-H. Yoon, I. Mochida, and J.-H. Chi, "Surface modification of carbon nanofiber with high degree of graphitization," Journal of Physical Chemistry B, vol. 108, no. 5, pp. 15331536, 2004

[5] T. Katayama, H. Araki, and K. Yoshino, "Multiwalled carbon nanotubes with bamboo-like structure and effects of heat treatment," Journal of Applied Physics, vol. 91, no. 10, pp. 6675-6678, 2002.
[6] N. A. Kiselev, J. Sloan, D. N. Zakharov, et al., "Carbon nanotubes from polyethylene precursors: structure and structural changes caused by thermal and chemical treatment revealed by HREM," Carbon, vol. 36, no. 7-8, pp. 1149-1157, 1998.

[7] J. Xu, J. P. Donohoe, and C. U. Pittman Jr., "Preparation, electrical and mechanical properties of vapor grown carbon fiber (VGCF)/vinyl ester composites," Composites Part A, vol. 35, no. 6, pp. 693-701, 2004.

[8] I. C. Finegan, G. G. Tibbetts, D. G. Glasgow, J.-M. Ting, and M. L. Lake, "Surface treatments for improving the mechanical properties of carbon nanofiber/thermoplastic composites," Journal of Materials Science, vol. 38, no. 16, pp. 3485-3490, 2003.

[9] R. J. Kuriger, M. K. Alam, D. P. Anderson, and R. L. Jacobsen, "Processing and characterization of aligned vapor grown carbon fiber reinforced polypropylene," Composites Part A, vol. 33, no. 1, pp. 53-62, 2002.

[10] M. Matzek, "Polymeric carbon nanocomposites: physical properties and osteoblast adhesion studies," M.S. thesis, University of Dayton, Dayton, Ohio, USA, 2004.

[11] M. L. Toebes, J. H. Bitter, A. Jos van Dillen, and K. P. de Jong, "Impact of the structure and reactivity of nickel particles on the catalytic growth of carbon nanofibers," Catalysis Today, vol. 76, no. 1, pp. 33-42, 2002. 

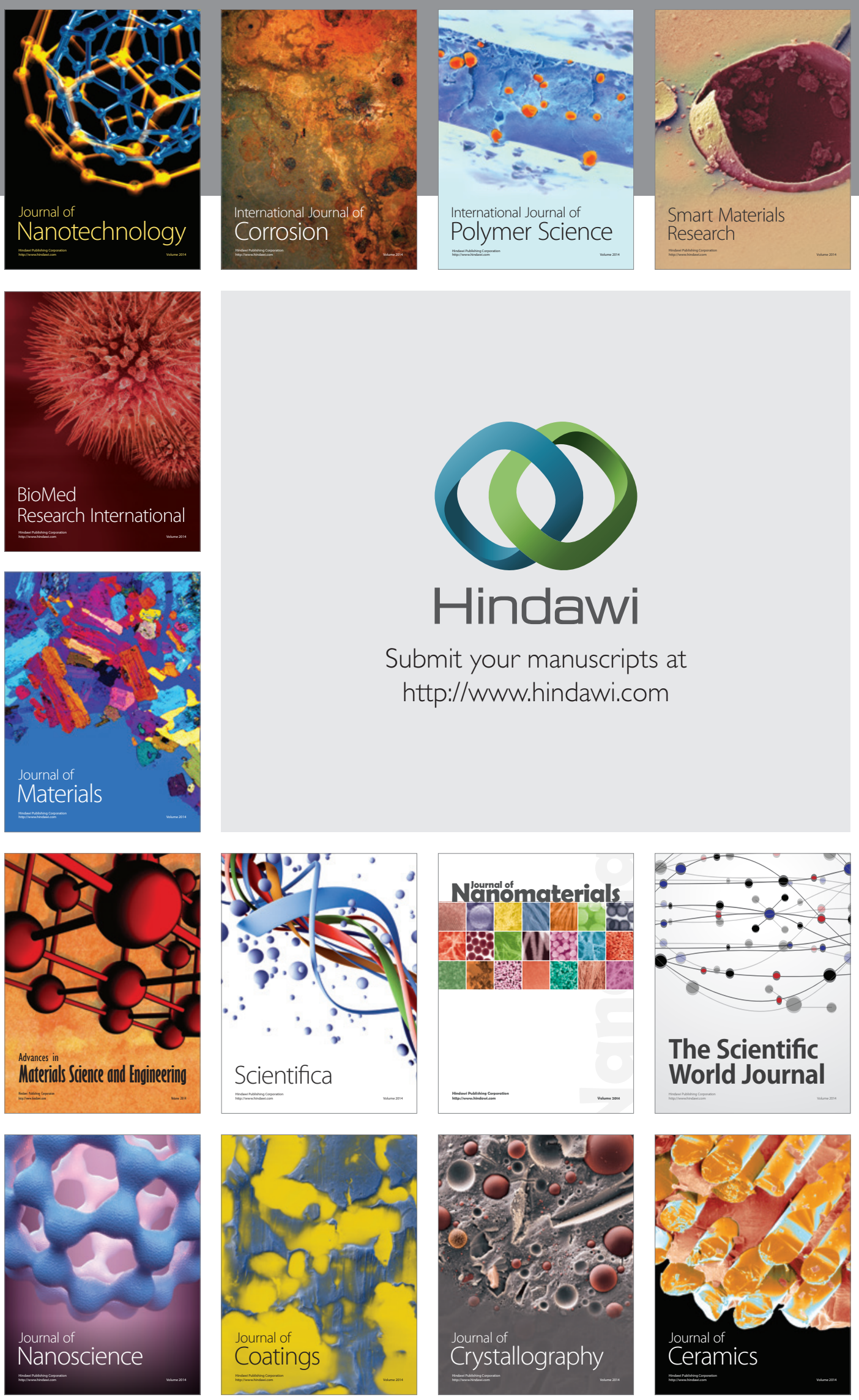

The Scientific World Journal

Submit your manuscripts at

http://www.hindawi.com

\section{World Journal}

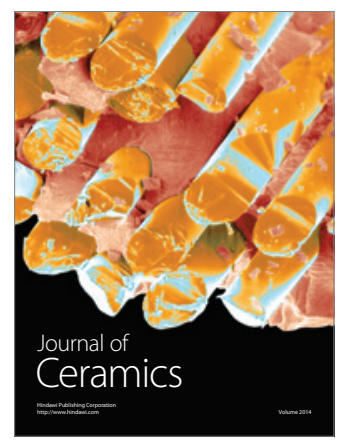

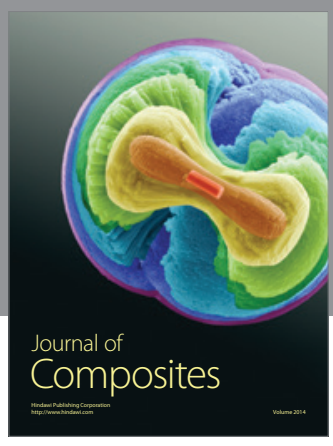
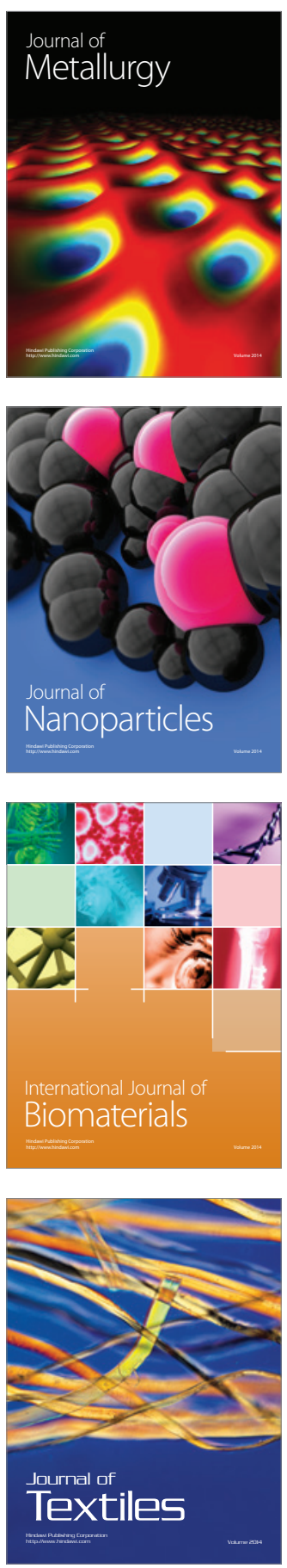